INTERNATIONAL DESIGN CONFERENCE - DESIGN 2018

https://doi.org/10.21278/idc.2018.0154

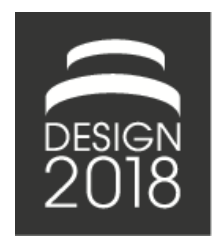

\title{
HUMAN CENTRICITY IN INTEGRATED DESIGN ENGINEERING
}

\author{
J. Urakami and S. Vajna
}

\begin{abstract}
Integrated Design Engineering is a holistic approach to create products of any kind. These are created in a human-centred way, as interests, issues, and needs of people involved in any product life cycle phase are respected. Attributes that don't fix realisation issues describe neutrally the required product performance. They result from needs, desires, expectations, and conditions in relation to the capabilities of users and from the environments where the product is created and to be used. Requirements to assure human centricity add to the requirements to realise the expected performance.
\end{abstract}

Keywords: human centricity, integrated design engineering, multi-/cross-/trans-disciplinary approaches, design strategy, product development

\section{Introduction}

The "Importance of People" has been rediscovered in recent years across various disciplines such as Economics, Architecture, and Engineering. This "Human Centricity" intents to integrate human characteristics by addressing questions such as "What do people want to accomplish? How do our actions affect people? How can we create value for people?". In the field of Engineering various approaches have evolved that put the human into the focus of both product development and production such as Design Thinking, Human-Factors Design, Human-centred Software Engineering, Engineering Psychology, and so on. However, the large variety of overlapping concepts is confusing and thus the difficulty for practitioners to choose the right concept for their needs and specific situations. Furthermore, the activities of conceptualisation, developing, designing, producing and distributing a product have become increasingly complex. This together with a high degree of labour division makes it necessary to cooperate and coordinate own activities with those of other participants in the product life cycle. Additionally, the continuing competition for natural resources and the highlighted awareness of how human activities affect the environment make it necessary to look beyond mere product development, production, and usage. In our perspective, Human Centricity is more than considering human characteristics in the emergence processes and the usage of a product, as it encompasses all aspects of human involvement and the (possible) effects on humans throughout the entire product life cycle.

The main focus of this contribution discusses the human centricity of IDE as the strongest driver for achieving a successful, reasonable, and sustainable product. Goal of this article is to provide a framework for current human centric approaches in design and engineering, to stipulate interdisciplinary work and to help researchers and practitioners to study and apply human centric design throughout various stages of the product life cycle. We first provide a representation of the life cycle of a product of any kind. Then, we describe the involved humans and their respective roles within the product life cycle in order to derive from these the different aspects and interdependencies 
of Human Centricity. Thirdly, as an appropriate framework for Human Centricity, we present Integrated Design Engineering (IDE), a holistic and comprehensive approach to develop products of any kind. All research work described here has been carried out and has been back-upped applying Insider Action Research according to (Björk, 2004) in more than 50 development projects with industry from different branches.

\section{The product life cycle}

The majority of concepts such as Human-Factor Design, Design Thinking or Human-centred software engineering focus on specific stages of the product life cycle such as development or usage. We believe that a holistic view of the product life cycle that encompasses all stages of a product from conceptualisation till recirculation (with the liquidation or the disposal of the leftovers as very last resort) is necessary in order to represent appropriately the complexity and interrelationships of real world events. Furthermore, many contemporary problems such as the increase of plastic in the oceans or the recycling and disposal of electronic products come from the short sightedness of focusing on specific stages of the product life cycle instead of a comprehensive holistic view. A holistic approach anticipates complex interrelations by involving all stages of the product life cycle.

The product life cycle includes the phases of product planning, product development, production, distribution, acquisition and product usage (including service and maintenance), as well as recirculation. In an ideal case the product life cycle is a closed cycle, e.g. physical components of products enter the life cycle of a new product after being refurbished or recycled. Immaterial objects such as software, methods, or procedures are replaced, re-used, or further developed and integrated into new software, methods, or procedures. Figure 1 provides detailed and self-explaining descriptions for each activity.

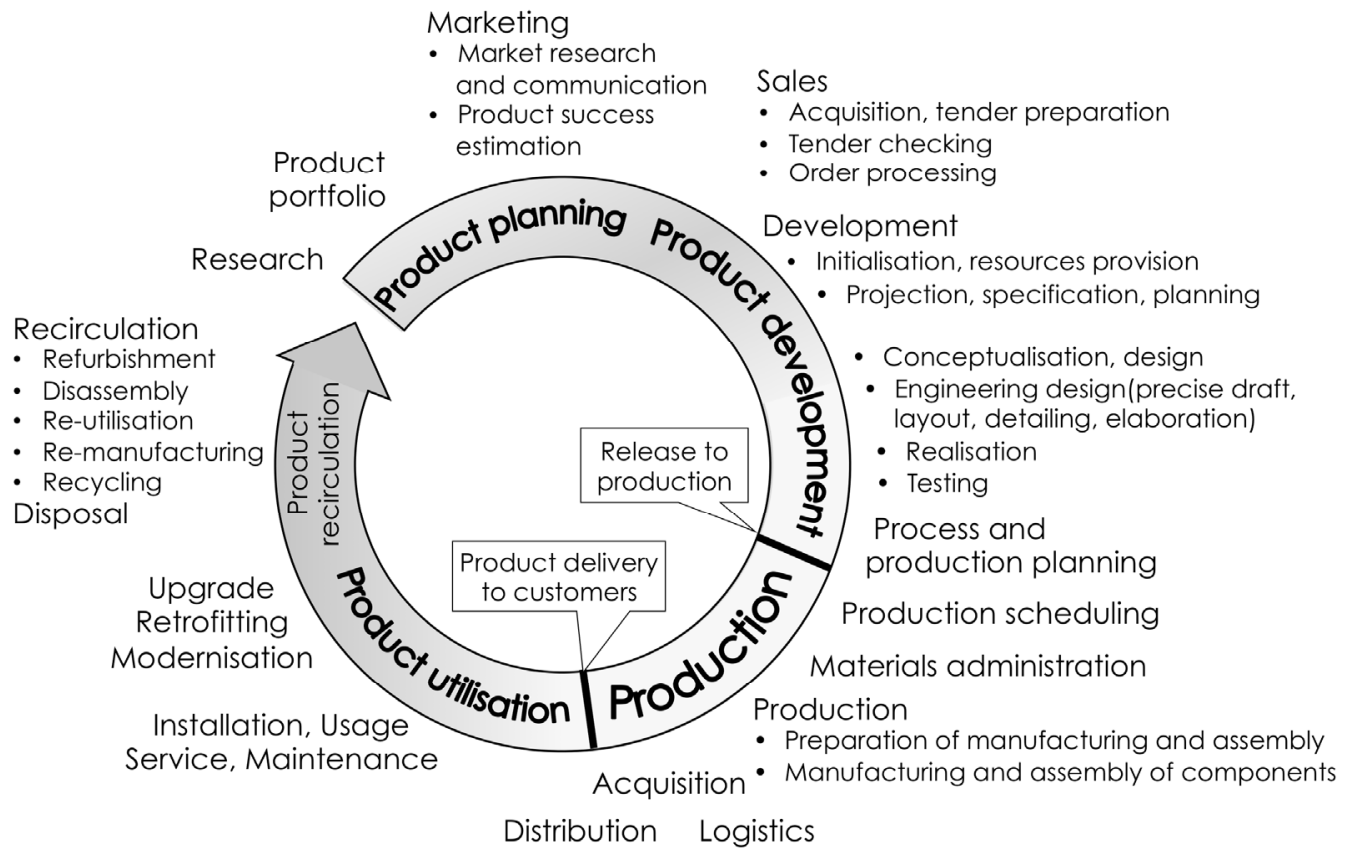

Figure 1. The product life cycle and its inherent activities

Besides the well-known distinction between product genesis and product usage, it is also possible to group the product life cycle by the following differentiators.

- Virtual vs. real environments: Product planning and product development run (with the exception of preparing and testing physical prototypes) in virtual environments, in which the emerging product is mainly and mostly represented in computer-based models. The transition between virtual environments and real / realisation environments happens at the release to production. Here, all 
documents that describe the product, its realisation, its utilisation, and its recirculation, have to be ready and consistent.

- Product ownership: The provider owns the product during the phases of product planning, product development, and production (including distribution). After its delivery the ownership is transferred to the customer for utilisation and maintenance.

- Resources utilisation vs. recirculation: At the end of the life of the product, it is put out of operation and transferred into the recirculation phase, consisting of e.g. reuse, refurbishment, upgrades, re-manufacturing disassembly, (re-utilisation and recycling, and, as last resort, disposal.

Furthermore, it is necessary to look beyond the product life cycle per se, thus to consider the interrelationship of the product and the contexts it exists within. According to our model, the product life cycle is influenced by four contexts, which have (1) cultural, (2) perceptual, (3) physical, and (4) regulatory characteristics. These contexts may affect all stages of the product life cycle as well as the humans involved in it. They may adapt to changes by e.g. general progress and by different social and political situations. In general, products interrelate in various ways with the contexts in which they are applied (Figure 2).

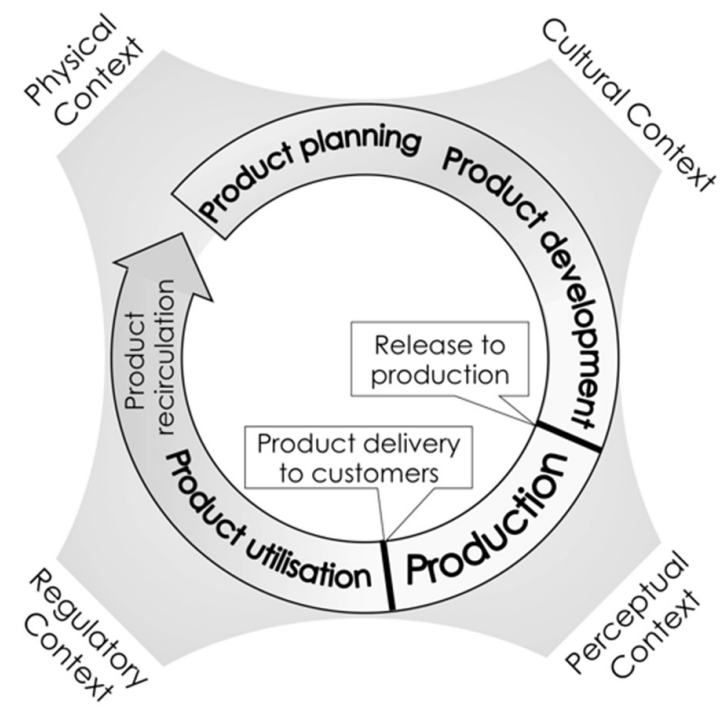

Figure 2. The product life cycle with influencing contexts

- The cultural context emerges from cultural values, among these life style, habits, communication patterns, working culture, or technological development of a culture. Cultural aspects affect the humans within the product life cycle, e.g. in the way of thinking (Nisbett, 2003), self-perception (Markus and Kitayama, 1991), and communication patterns, beliefs, and value systems. As a consequence, products have to represent the cultural values of their targeted users. Products can also affect cultural elements, e.g. smart phones or text messaging have broadened the ways of communication and exchanging ideas.

- Perceptual context refers to how people perceive and comprehend the world around them. People from different cultural backgrounds perceive the world differently and process information in a different way (e.g. Nisbett, 2003). Providers and customers with different cultural backgrounds might differ in their perceptions and attitudes towards products. Cultural differences exist in regard to information processing, stereotyping, and categorization when and how products are used.

- The physical context refers to the geographical and psychological location in which a product is developed and used. The physical context affects the design of products to ensure their reliability. The physical environment and the product are mutually affected by the product application or its side effects e.g. exhaust fumes of cars causing air pollution.

- The regulatory context covers laws, regulations, standards, and guidelines. These govern the genesis, the application, and the recycling of a product in its target environments on different levels 
of strictness. They provide a knowledge base for manifold best practices thus offering guidance when developing, manufacturing, and using solutions of any kind. On the one hand they help to minimise aberrations, on the other hand they may hinder innovations or breakthroughs, e.g. selfdriving cars may require new regulations and guidelines about manufacturing issues as well as insurance policies or responsibilities in case of an accident.

\section{Groups of humans involved in the product life cycle}

When talking of humans within the product life cycle, two groups that are involved at and directly affected by different states of the product life cycle are obvious, the customers and the providers. Customers and providers each have their own intentions, goals, and interests connected to a certain product and its realisation and utilisation processes, thus can be called the stakeholders.

\subsection{The customer}

The general (and main) target for setting up a product and starting its life cycle is the intention to satisfy demands of humans. Demands result as a synthesis from different customer needs and necessities, desires, and (partly hidden) expectations in relation to their capabilities and limitations, as well as from the conditions of the four contexts in which the product is to be used (Ottosson, 2013). Demands come from a certain customer, who may be a single person, a group of persons, or institutions within specific markets. Each customer defines a different business, has different expectations and values, and buys something different. A customer may be a user, or a buyer, a sponsor, a patron, or combinations of these (mainly of user and of buyer). These humans may be involved with the conditions for product existence in a given or changing environment (legal, social, etc.). The following subchapters explain briefly the intentions, goals, and interests of these groups.

\subsubsection{The user}

The user is the person or a group of persons for whom the product is aimed at and designed for, the person, who ultimately uses or intents to use the product. The user wants a product to achieve his specific demands effectively, efficiently, and satisfactorily. Therefore, in developing the product, special consideration have to be given also to the capabilities and limitations of the user. As mentioned above, this consideration leads to additional requirements besides those for product performance and product behaviour. The user should not be forced to adjust or to adapt to the given capabilities of the product, but the product should be fitted to the user's needs, abilities, and qualifications.

Different approaches to capture the demands of users (in the broadest sense) in order to better focus on these have been researched, among these e.g. Usability and User Experience that both focus mainly on the usage phase. For assuring this focusing on humans, the following criteria apply, which are realisation, protection from harm, building on capabilities, and personal development, Table 1.

Table 1. Criteria for focusing on humans

\begin{tabular}{|l|l|l|}
\cline { 2 - 3 } \multicolumn{1}{c|}{} & Provider & User \\
\hline Realisation & $\begin{array}{l}\text { The provider has the necessary skills, resources, knowledge, } \\
\text { capabilities to realise the product }\end{array}$ & $\begin{array}{l}\text { Basic foundations of human perception, cognition, and motor control are } \\
\text { considered already in the design. The user can perceive and understand } \\
\text { information and is able to carry out operations }\end{array}$ \\
\hline $\begin{array}{l}\text { Protection } \\
\text { from harm }\end{array}$ & $\begin{array}{l}\text { Humans working in the product life cycle are protected from } \\
\text { impairment, dangerous substances, in-appropriate working } \\
\text { conditions, etc. They work in a reasonable environment. } \\
\text { Human capabilities and limitations are not overstrained }\end{array}$ & $\begin{array}{l}\text { The user is protected from impairment. User's capabilities and limitations are } \\
\text { not overstrained }\end{array}$ \\
\hline $\begin{array}{l}\text { Building on } \\
\text { capabilities }\end{array}$ & $\begin{array}{l}\text { Being able to apply knowledge, abilities, and skills in the work } \\
\text { process }\end{array}$ & Building on users capabilities, knowledge, expectations, and skills \\
\hline $\begin{array}{l}\text { Personal } \\
\text { development }\end{array}$ & $\begin{array}{l}\text { Developing new skills, learning something new, thus gaining } \\
\text { new knowledge and experiences in the work process }\end{array}$ & $\begin{array}{l}\text { The product stimulates new experiences, helps to improve skills, and } \\
\text { increases knowledge }\end{array}$ \\
\hline
\end{tabular}

Thus, the requirements for assuring human centricity add in every respect to the requirements resulting from the expected product performance. When exploring these, possible reciprocal influences have to 
be taken into account and balanced out. However, these effects are more than counterbalanced by focussing on humans, amongst others by much better focus on target groups, higher acceptance, and less consumption of resources during product genesis, usage, and recirculation.

\subsubsection{The buyer}

The buyer is a person or group of persons who purchases the product. If a buyer purchases the product for his/her own use, then buyer and user are the same person. However, if the buyer is only responsible for the acquisition and the payment of the product, then buyer and user are different persons or institutions, and they pursue different goals. The buyer is e.g. looking for low prices, for a simple and durable product without problems for many different uses, where (almost) no maintenance is required. This may lead to a conflict of interests between the buyer and the user. As a consequence, the different target groups of a product have to be clarified and to be taken into account from the very beginning of product development, even if they may contradict each other. For example, a health insurance company (the buyer) provides a wheelchair to a handicapped person (the user). The health insurance company wants a cheap, durable, and robust device that may be used by different parties. The user would appreciate a customized chair that is comfortable, not too heavy, and that can be pushed easily.

\subsubsection{The sponsor and the patron}

Sponsor and patron are special kinds of buyers. A sponsor links his own message to a product by using or enforcing the prestige of this product, and therefore pays the product for e.g. a user. A patron may support customers and/or providers by allocating financial, organisational, technical, or ideational means to the acquisition and / or genesis of a product. The reasons for patronage may range from philanthropy to concrete economic and / or political interests.

\subsection{The provider}

The denomination "provider" is derived from the customer's point of view. Customers get products that are, from the customer's view, provided from a more or less anonymous and not always transparent institution, of which the inner structure normally is not in the focus of interest of the customer. This term is used here to cover all possible persons, group of persons or institutions that, within institutions, contribute to the planning and the realisation of a product, its transition to the consumer, its service / update, and its recirculation at the end of the product life cycle. Such institutions could be enterprises, manufacturers, agents, retailers, suppliers, distributors, etc.

- An enterprise covers all functions and activities necessary to conceptualize, to generate, to deliver products to customers and servicing them, and to take care of their recirculation at the end of its life cycle.

- The manufacturer as an independent company is responsible for the production that is triggered by the input of an external ordering institution, i.e. is the supplier for this institution.

- An agent acts as an intermediary between customers and enterprises, retailers, and distributors, whereby the activities of the agent covers both directions between customers and institutions.

\section{Foundations of Integrated Design Engineering}

IDE synthesizes and integrates elements of IPD, Dynamic Product Development, User-centred Design, Sustainable Design, Design for X (DfX considers influences from the domains " $\mathrm{X}$ " on product development, whereby " $\mathrm{X}$ " is a place-holder for e.g. manufacturing or assembly), as well as Industrial and Organizational Psychology, thus acting in a much greater range of application than a simple summation of the areas of the mentioned approaches. In IDE, products of any kind are described and developed as well as changed and adapted based on multi-criteria requirements from both the product target groups, on all phases of the product life cycle, and on the humans involved in this life cycle. IDE is therefore human centred, uses multiple criteria to describe product performance, fosters interdisciplinary work, and applies a holistic view (Vajna, 2014).

Components of IDE are the necessary means and procedures for human centricity, the description of a product with attributes, the various types of integration, and the underlying holistic procedure model. 
Products in IDE may be physical objects (discrete or continuous) such as mobile phones, TV sets, bicycles, or immaterial objects such as software, services, teaching, research, methods, procedures, or (almost) any combinations of these. IDE takes into account the whole product life cycle. It is based on eleven different types of integrations, at first the integration of all humans or parties involved, then products, processes, departments, knowledge, and methods, respectively.

Within IDE, customer demands (see chapter 3.1) are translated into requirements for a product in the broadest sense. To cover and to fulfil these requirements in a neutral way without pre-fixing realisation strategies and means, a product is described by its performance, which is defined here as combination and interaction of the capabilities of a product and the possibilities to put these capabilities into utilisation (i.e. the behaviour of the product). This performance is modelled by eleven equal but not identical attributes resulting from the product life cycle, both from the view of the customer and from the view of the provider. The attributes also consider the humans involved in the life cycle (provider, customer, persons affected), thus allowing a neutral product description without determining realisation strategies before maturity (Vajna, 2015).

- From the customer's view the attributes describe the customers' demands to product performance. Attributes from the customers view form the target attribute profile.

- From the provider's view the attributes describe the performance the product offers. Especially in the consumer good markets, the offer can also be influenced in the broadest sense by regulations and guidelines based on various customer requirements, by emerging market segments, or conditions of the product environment. Attributes from the provider's view form the as-is attribute profile.

There are six product attributes, three fulfilment attributes, and two economic attributes.

- The first three product attributes are Product Gestalt (How does it look like? What perceivable information is offered?), Functionality (What can it do?), and Usability (Is it easy to use? Is using it satisfactory?). The synthesis of these three attributes is summarised as Suitability. The fourth attribute is two-sided. From the provider's view it is Producibility (Is it possible to make?), from the customer's view it is Availability (Is it gettable when requested or needed?). The last two product attributes are Maintainability (Is it easy to upkeep?), and Sustainability (Is the negative impact on the environment low?).

- Fulfilment attributes are Safety (Is the user protected from harm?), Reliability (Does it provide its required performance over the expected usage time?), and Quality (Does it fulfil user demands with the anticipated level of excellence?).

- Economic attributes are Added Value (customers' view: What can I get for my money, including ideational values?) and Return on Investment (provider's view: Can I make a profit?).

The underlying IDE procedure model provides, on the top level, eleven activities to create and to develop a product. These activities form self-similar patterns on any level of concept, specification, and realisation. The eleven activities are at first researching, then developing, designing, and integrating, followed by modelling, configuring, and synthesizing in order to concretise concepts, layouts, and approaches. Detail work is assured by the completing activity. All these activities are assessed and supervised by evaluation, comparison, and selection. Lower layers of activities provide practical approaches, procedures, methods, and tools for the activity in question. There is not a prescribed sequence of activities in this model. In fact, the activities are event-driven, as they are processed dynamically according to the actual state of product emergence in context with the actual state of requirements to the performance of the product (Vajna, 2016).

\section{Human Centricity in IDE}

One central aspect of IDE is the focus on all humans within the product life cycle. We differentiate between three main groups of humans that are involved at different states of the product life cycle: Customers and providers (as discussed in chapter 3), and furthermore persons concerned. Taking into account the group of persons concerned as third equivalent group of humans involved, is one of the main differences of IDE to other development approaches. 
Persons concerned don't have any intention and interest in dealing with the same product as customers and providers. On the contrary, creation, application, and recirculation of the product can hinder persons concerned in pursuing their own notions in their own environments and contexts.

Users and providers have of course the strongest effect on the creation of products and are indispensable in the product life cycle, thus can be named as stakeholders. The provider ensures that an idea is turned into a product and is realised. The user is not only utilising the product but also ensures that the product serves its purpose and fulfils its creation objectives. However, both have to respect the necessities and expectations of the persons concerned.

Hence, human centricity in IDE is defined as both respect for and consideration of all interests, issues, needs, and matters of humans involved with a product throughout its whole life cycle. It means, as a product is generated, distributed, used, serviced, re-utilised in ways with the appropriate means and processes, that anyone dealing with the product in any of its life phases or is affected won't be overstrained in regards to his perception capabilities, and personal limitations. Nor won't they suffer any harm, and won't need to work or to live in hazardous or unethical environments, Figure 3.

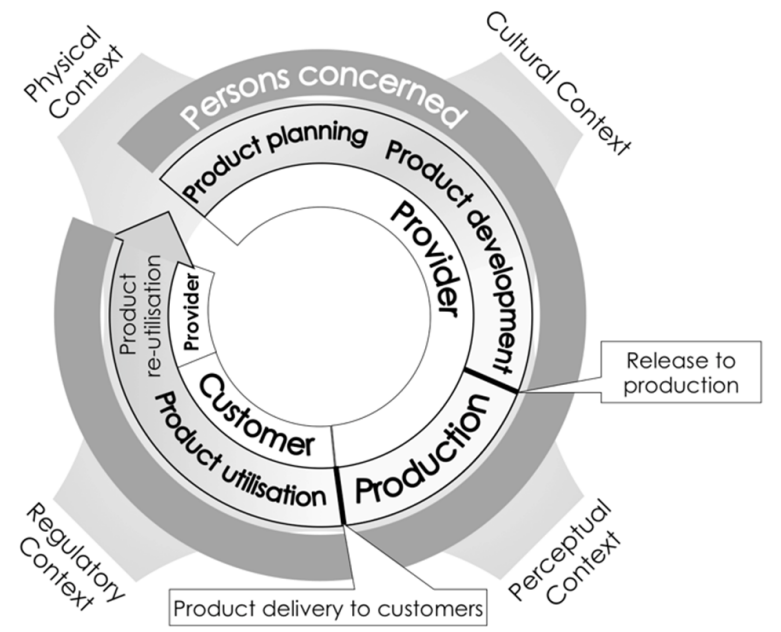

Figure 3. Different groups of humans along the product life cycle within IDE

The Figure shows clearly that Human Centricity within IDE goes far beyond the various explicit and implicit considerations of the user alone, as a user-focussed view only highlights the product utilisation phase. Summarizing it can be said, that Human Centricity in IDE supports the product experience of the humans covering the complete product life cycle, i.e. it is not only focussed on the user of the product. The human centricity of IDE covers more than the approaches of Usability and User Experience that both focus mainly on the usage phase.

Required for the development of successful products (and in addition to the proven and known approaches for problem solving) are, in general, spontaneity (a sudden action due to a flash of inspiration or an insight), intuition (immediate inspiration without a perception process), and creativity (imagination richness, creative power). Machines cannot yet model these three typical human characteristics. Thus, IDE can only exist with humans as creative problem solvers, because only they with their multidimensional qualification based on knowledge, experience, competence, and skills are able to acquire and generate knowledge. The human is the only one to be able to filter information from data, to creatively apply knowledge as well as to provide and to pass on appropriate and suitable information. These ideas have been already emphasized at the beginning of the $20^{\text {th }}$ century by Lillian M. Gilbreth and Frank B. Gilbreth, who were one of the firsts to acknowledge the crucial role of human centricity (Graham, 1998). They combined insights from the management of a company with findings from engineering sciences and work psychology in order to create a different view on the interdependencies between employee performance and company's working environments. In particular, they appreciated workers as individuals and did not regard them, as in other contemporary approaches, as a simple production resource, equivalent to and replaceable by machines, materials, and money. 
Similarly, Shunk (1988) stated that the success of a company is composed of $10 \%$ hardware (i.e. factory equipment, machine tools, office equipment, computer hardware), $40 \%$ software (procedures, guidelines, software used, etc.), and 50\% "peopleware", i.e. multiple-qualified employees, the so-called knowledge workers. These prefer to work in "learning companies" where work in general is regarded to be a continuous qualification (Speck and Kees, 1994). Such people are, among others, able to react flexibly and appropriately to unforeseen disturbances or changes of customer demands.

The competency profile of humans within IDE includes professional, social, and personal skills as well as entrepreneurial skills (Berliner Kreis, 1997; Hofer, 2010).

- Professional competence is based on the expertise that includes all knowledge, capabilities, and skills necessary to accomplish the development tasks.

- Social competence concerns all characteristics that facilitate and simplify co-existence and cooperative work in groups and teams. This also includes appropriate communication behaviour and the ability to settle conflicts without reciprocal personal harm.

- Personal competence is expressed in the correct attitude (e.g. responsibility, goal-oriented action, self-organization), in independent and holistic thinking (manifested in a timely and complete assessment of the effects of one's own actions), in the personal value system, in a realistic selfassessment, and the motivation to problem solving.

- Creative skills enable humans to form something new and valuable.

- Entrepreneurial skills encompass the will, the commitment, and the ability to directly contribute to the success of the (own) company, together with the realistic estimation of possibilities and risks of the enterprise in the market.

IDE thus creates an environment in which independent, self-responsible, active, and content people work. In this environment, the work maxim is to do the right tasks and the tasks right, both at the right time and place, instead of having to repair them later. Hence, integral parts of human centricity are:

- Humans work in both homogenous and multidisciplinary teams on all decisive activities and phases. These teams are able to plan, to design, and to implement holistically appropriate results for all phases of the product life cycle (of course, special and clearly defined tasks can also be carried out by a sub-team within a team or by individuals). IDE leads to a co-operative development not only in the company itself but also with customers, partners, and suppliers, or associations and authorities. Only in such a way it is possible to achieve convincing and innovative products.

- It comes to higher self-responsibility with greater decision-making potential in the sense of entrepreneurial action, with a strong participation of employees in the design of work tasks, taking into consideration the abilities, characteristics, creativity, and predisposition of each employee.

- Employees and executives work together inter-disciplinarily and in partnership, maintain an open communication and information exchange with quick and short feedback. Leadership is affected by delegation of responsibilities and coaching, not necessarily by supervision and control.

- In order to provide solutions at any time, which incorporate the current state of knowledge, there is the need for a sufficient amount of time for continuous qualification (in the sense of lifelong learning). Much of this can be achieved through multidisciplinary project work.

- Since IDE may be carried out within a company as well as in trans-frontier company networks, it is influenced by different ethical and cultural values and peculiarities of the humans involved.

- Only such methods, procedures, and tools are used, in which human thought, capabilities, and action are adequately taken into account. Systems and programs should have highly user-friendly interfaces (e.g. dynamically adapted command offer, intuitive usage possibilities, dynamic user management, robust error tolerance, etc.), which do not bother the staff.

The integration of different areas of activity results in increased self-reliance and an on-going adaptation of qualifications to an improved ability to learn. The predominant teamwork leads to a steady improvement in the ability to co-operate, all of which lead to a high level of self-esteem.

In general, the requirements for assuring human centricity add to the requirements resulting from the expected product performance. In the following, user, provider, and persons concerned are described in detail in the view of IDE. 


\subsection{The user}

The IDE approach to describe and to model the demanded (user's view) or rather the possible product performance (provider's view) by attributes fosters the accommodation of a product to a wide range of users with a large variety of capabilities and limitations.

Based on how human orientate themselves and act in their living environment, three basic needs of the user can be derived as requirements for product design that are 1) perceive, 2) comprehend, and 3) respond/operate (Figure 4).

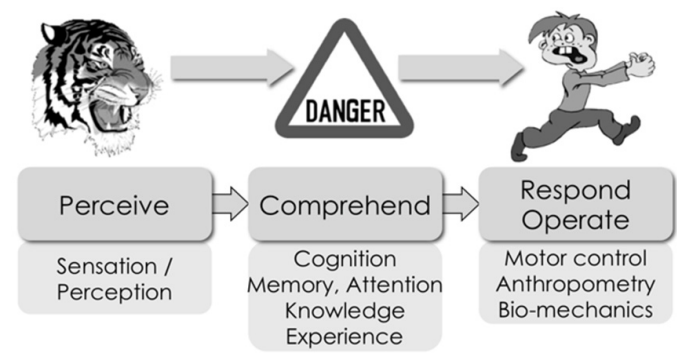

Figure 4. How humans perceive, comprehend and respond to environmental information

- Perceive: There are two different dimensions in perceiving that are important for product design, the aesthetic dimension and the semantic dimension (Zeh, 2010). Within the aesthetic dimension it is important that the product offers information that is most appropriate to the target user group. This must convey an optimal level of stimulation in order to experience a positive affect (Zeh, 2010). In addition, it is also important to design a product in a most advanced but yet acceptable way (Loewy, 1951), so that there is a well-balanced level between familiarity and newness in the design of the product (Zeh, 2010). Within the semantic dimension the user must be able to perceive information provided by the product. The user has to be able to find controls, perceive displayed information, feedback, and the system status. This implicit information within the product about its usage possibilities is known as Affordance (Gibson, 1977). Out of the five senses (visual, auditory, haptic, olfactory, gustatory), product design usually refers to the first three senses, visual, auditory, and haptic, to provide information for the user. Basics of human sensation and perception such as the minimal threshold for perceiving sensory input (the minimum magnitude of a stimulus that can be discriminated from no stimulus at all) and just noticeable difference (minimum stimulus magnitude to tell two stimuli apart) have to be respected in product design.

- Comprehend: The user must be able to comprehend the perceived information, process it, interpret and evaluate it. Users' capabilities and limitations of cognitive processes such as memory, attention, learning, problem solving, remembering, and language have to be considered in product design and thus are mandatory in IDE. The user has to understand how to use the product. Gestalt principles have to be considered to classify information in a way that works well for human cognitive processes. Because of limitations in his memory capacity, the user should not be required to hold information for too long time in working memory. Sequencing the presentation of information is preferred in order to reduce complexity. Furthermore, users' knowledge and experience have to be considered as well. Design criteria of Cognitive Ergonomics provide guidelines for increasing the comprehensibility of interfaces that are compatibility, consistency, individualization, robustness (error tolerance), transparency, learnability, memorability, efficiency and satisfaction.

- Respond/Operate: The user must be able to respond appropriately to the information provided by the product. The user must be able to operate the product safely and to carry out all functions at the expected levels. Information from the product as well as from the user's body is integrated to generate a desired motion or action. The physical dimensions of the product have to be matched to the physical dimensions (anthropometry) of the user's body to design clearance, grips, and positions of displays and controls, respectively. Capabilities and limitations of the user's motor control that is the coordination of body movements and muscle strength (biomechanics), precision 
of movements, and time needed to carry out specific actions have to be considered in product design.

\subsection{The provider}

Within IDE, providers are composed of humans realising the tasks mentioned in Figure 1. Some of their roles may be overlapping and the same person can be involved in different roles at different stages of the product life cycle.

Within a company, human centricity results in a change in both approach and co-operation of daily tasks, which has a positive effect on the motivation, the innovative power, and the creativity potential of humans involved. Thus, if the company acknowledges the importance of its employees, this has a beneficial effect on corporate culture and, consequently, on the motivation, innovativeness, and creativity potential of its employees.

Means of human centricity are personnel development, a motivation-oriented management, and the introduction of the continuous improvement process of products and development processes (Hofer, 2010). In IDE, the appropriate and confidence-building personnel development plays the essential role. Such a development that is both wanted by the human himself (as an intrinsic motivation) as well as required and promoted by the company (as an extrinsic motivation) results in the further development of the competence profile that leads to and fosters a human-centred, responsible, integrated, interdisciplinary, and holistic way of working in IDE.

Thus IDE is in agreement with similar ideas in modern Human Resource Management (HRM) approaches (see Aamodt (2015) for an overview). Employees in an organisation are treated as key resources and not managed as factors of production. Employees are seen as crucial investment for the organisation. Fulfilling the needs of employees and encouraging them to apply and to develop their skills are central elements of HRM. HRM proposes that higher employee motivation and the opportunity to apply skills and abilities will improve quality and quantity of work, resulting in higher organizational productivity and profits that enable greater employee rewards and recognition, further boosting employees' motivation.

\subsection{Persons concerned}

Persons or groups of persons concerned are part of the environments in which a product is embedded throughout its whole life cycle. Persons concerned are often overlooked in other approaches but are treated here as an important third party that needs to be considered in the product life cycle.

- The development and (mainly) the production of a product can affect persons in the physical environment of the enterprise in which the product is developed, manufactured, assembled, and serviced. The affection may range from noise annoyance by production processes, over to pollution of the environment, and end in traffic disturbances caused by transportation operations. Many companies produce their goods in $3^{\text {rd }}$ world countries for the benefit of lower wages thereby causing tremendous environmental pollution.

- The utilisation of a product in a certain environment can affect or disturb directly persons in the same environment in various ways, e.g. when one is bothered by compulsory listening to manmade noise created by loud music coming of a neighbour in a train. Another example is a leaf blower that might be beneficial for the user because it is less work than sweeping leafs with a broom, but it works with noise exposure and produces exhaust fumes. Thus, persons could be disturbed who have an indirect connection to the product or process.

- The recirculation of a product can disturb persons and their respective environments by various impacts when disassembling, re-assembling, recycling, and disposing the product, e.g. when toxic gas escapes during the recycling of electronic goods that threatens the people working in recycling and people living in this environment.

Although laws and regulations are trying to reduce the negative impact of certain products on affected persons by regulating product usage (like smoking prohibitions in public buildings or restaurants), product development has to strive for reducing negative impacts on persons concerned as much as possible. To achieve this, the consideration of the four contexts in which the product will be embedded 
(c.f. Figure 3) is of high importance throughout the development of the product. For example, catalysers in cars do not improve the performance of the car but reduce its negative impact on persons concerned by less polluting the air.

\section{Conclusion: Theoretical and practical implications of IDE}

The main characteristic of IDE is the focus on human centricity, which is defined as both respect and consideration of all interests, issues, needs, and matters of all humans involved with a product throughout its whole life cycle. It has been shown that human centricity is the strongest driver to achieve a successful, reasonable, and sustainable product. As a consequence, a product has to be generated, distributed, used, serviced, re-utilised in ways with appropriate processes. It also means that anyone dealing with the product in any of its life phases won't be overstrained in regards to his capabilities and limitations, won't suffer any harm, and won't need to work in hazardous or unethical environments.

The requirements for assuring human centricity must be added to the requirements resulting from the expected product performance. Thus, current and future research on human centricity in IDE is performed on comprehensive means and approaches to capture and to model the resulting requirements as addition to the requirements for product performance, and to map them also on the IDE attributes. Applying IDE principles throughout the product life cycle is beneficial for producers, end-user, $3^{\text {rd }}$ parties and people who work in the product life cycle. IDE helps to specify characteristics of the product that encompass the entire product life cycle of designing, production, distribution, usage, and recirculation. It supports the producer in the design process to decide product specifications, e.g. product gestalt, functionality, safety etc. IDE is a useful tool for product planning. Product attributes in IDE can be used as a checklist to ensure that the product will perform well across the entire product life cycle. Furthermore, IDE can serve as a tool for evaluating the human centricity of a product. Compared to existing concepts such as Usability evaluation, IDE looks beyond the usage phase of the product and also considers whether human centric criteria are regarded for people who work in IDE, such as developers, producers, distributors and recyclers. Additionally, the impact of the product on humans concerned is considered as well. This aspect is not reflected in previous human centric approaches.

In research, IDE can function as a model to describe, explain, and predict the life cycle of products. IDE can be used to understand the impact of products, product development, usage and re-utilization on humans who are directly or indirectly involved in the design process. IDE helps to identify influencing environments and to determine interrelated effects between product life cycle and environmental aspects such as physical context, cultural context, and regulatory context such as laws, standards and guidelines. Thus IDE serves as a framework for interdisciplinary research looking at relationships of HumanMachine interaction, Technology and Culture/Technology and Society.

IDE can also be used to educate customers about the life cycle of products in order to influence purchasing habits of customers. Customers usually look at a product in its "present" state (Do I like what I see?), but do not consider the products past (development and production process), and the products future (possibility of re-utilization or recycling). If we want to create a sustainable society that uses its resources wisely then we have to educate customers and make the impact of the product life cycle on humans and environment visible.

From the provider's perspective, IDE helps to predict how a product moves through its life cycle and it connects different parties involved in that process. Thus, IDE can be used as a tool to enhance and promote communication between designer and developer, people involved in production, sale, and recycling, as well as with the costumer. Highlighting the product's life cycle increases the visibility of the connection between different parties involved and might increase costumers' trust in a product. This is especially important for certain types of products such as food. IDE can also support a company's effort to implement quality management as described in ISO 9001, by a better coordination of stakeholders and improved communication between involved parties. Furthermore, a responsible use of resources, being it raw materials, energy or human resources, as well as a conscious awareness of the products impact on its environment throughout its life cycle reflects on the ethical values of a company and its reputation. Additionally, understanding local differences in the requirements of the physical, cultural, perceptual, and regulatory context a product exists within enables companies to adapt their products successfully to local markets. 
Human centricity is about all aspects of human involvement and effects throughout the entire product life cycle including humans that provide the product, use the product, and humans that are affected by the product indirectly. In this article we introduced a holistic theory of Integrated Design Engineering with the goal to provide a framework for current human centric approaches in design and engineering, to stipulate interdisciplinary work and to help researchers and practitioners to study and apply human centric design throughout various stages of the product life cycle.

\section{Acknowledgement}

The authors would like to thank Industrial Designer Martin Wiesner M.A. for providing beneficial knowledge and experience in working with the Product Gestalt.

\section{References}

Aamodt, M.G. (2015), Industrial/Organizational Psychology: An Applied Approach ( $8^{\text {th }}$ Edition), Cengage Learning, Boston.

Berliner Kreis (1977), Neue Wege zur Produktentwicklung - Untersuchung im Rahmenkonzept «Produktion 2000», Projektträgerschaft: Fertigungstechnik und Qualitätssicherung, Forschungszentrum Karlsruhe GmbH, Universität-GH Paderborn, Heinz Nixdorf Institut, Paderborn.

Björk, E. (2004), A Contribution to Insider Action Research Applied on the Development of Assistive Products, Book series "Integrated Product Development", Magdeburg.

Gibson, J.J. (1977), “The Theory of Affordances”, In: Shaw, R. and Bransford, J. (Eds.), Perceiving, Acting, and Knowing, John Wiley \& Sons Inc., Hoboken, NJ, pp. 127-143.

Graham, L. (1998), Managing on her own: Dr. Lillian Gilbreth and women's work in the interwar area, Engineering \& Management Press, Norcross.

Hofer, M. (2010), Anwendungsgebiete von Coaching als Methode zur Förderung der Humanzentrierung in Unternehmen, Master thesis, Hochschule Mittweida.

Loewy, R. (1951), Never leave well enough alone, The Johns Hopkins University Press.

Markus, H.R. and Kitayama, S. (1991), "Culture and the self: Implications for cognition, emotion, and motivation", Psychological Review, Vol. 98 No. 2, pp. 224-253.

Nisbett, R.E. (2003), The geography of thought: How Asians and Westerners think differently and why, Free Press, New York.

Ottosson, S. (2013), Frontline Innovation Management, 2nd ed., Tervix Göteborg.

Shunk, D. (1988), "CIM in den USA”, Fortschrittliche Betriebsführung/Industrial Engineering, Vol. 37 No. 1, pp. 19-25.

Speck, P. and Kees, U. (1994), “Auf dem Weg zum Lernunternehmen - Beispiel Werk Rohrbach der FESTO KG” Personalführung, Vol. 7, pp. 600-607.

Vajna, S. (2014), Integrated Design Engineering - Interdisziplinäres Modell für die ganzheitliche Produktentwicklung, Springer, Heidelberg.

Vajna, S. (2016), “The Holistic Procedure Model of Integrated Design Engineering”, The Publications of the MultiScience - XXX. microCAD International Multidisciplinary Scientific Conference, Miskolc, Hungary, April 21-22, 2016, University of Miskolc, Miskolc.

Vajna, S. (2015), “Attributes in Integrated Design Engineering - a new way to describe both performance capability and behaviour of a product", Proceedings of the 20th International Conference on Engineering Design (ICED 15) Volume 2: Design Theory and Research Methodology, The Design Society, Glasgow, pp. 127-136. Zeh, N. (2010), Erfolgsfaktor Produktdesign, Fördergesellschaft Produkt-Marketing, Köln.

Univ.-Prof. Dr.-Ing. Prof. h.c. Dr. h.c. Sandor Vajna

Otto von Guericke University Magdeburg

POB 4120, D-39016 Magdeburg, Germany

Email: vajna@ovgu.de 